\title{
Los pastizales y matorrales de zonas áridas y semiáridas de México: Estatus actual, retos y perspectivas
}

\author{
Pedro Jurado-Guerra ${ }^{a^{*}}$ \\ Mauricio Velázquez-Martínez ${ }^{\text {b }}$ \\ Ricardo Alonso Sánchez-Gutiérrez ${ }^{\mathrm{c}}$ \\ Alan Álvarez-Holguín a \\ Pablo Alfredo Domínguez-Martínez ${ }^{\mathrm{d}}$ \\ Ramón Gutiérrez-Luna ${ }^{c}$ \\ Rubén Darío Garza-Cedillo ${ }^{\mathrm{e}}$ \\ Miguel Luna-Luna ${ }^{f}$ \\ Manuel Gustavo Chávez-Ruiz ${ }^{\text {a }}$
}

${ }^{a}$ Instituto Nacional de Investigaciones Forestales Agrícolas y Pecuarias (INIFAP). Centro de Investigación Regional Norte-Centro (CIRNOC), Campo Experimental (CE) La Campana, Km. 33.5 Carretera Chihuahua-Ojinaga, Aldama, Chihuahua, 32910, México.

${ }^{\mathrm{b}}$ INIFAP, CIRNE, CE San Luis, San Luis Potosí, México.

${ }^{\mathrm{c}}$ INIFAP, CIRNOC, CE Zacatecas, Zacatecas, México.

${ }^{\mathrm{d}}$ INIFAP, CIRNOC, CE Valle del Guadiana, Durango, México.

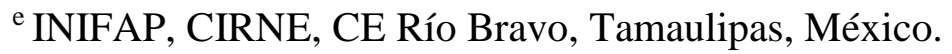

${ }^{\mathrm{f}}$ INIFAP, Centro Nacional de Investigación en Agricultura Familiar, Ojuelos, Jalisco, México.

* Autor de correspondencia: jurado.pedro@inifap.gob.mx 


\section{Resumen:}

El objetivo fue revisar el estado actual de los pastizales semiáridos y matorrales áridos del norte y centro de México, así como analizar los retos y perspectivas del uso de estos ecosistemas. Desde la década de los años 1950, el INIFAP en colaboración con otras instituciones han generado y transferido conocimientos sobre manejo de pastizales, lo cual se ha reflejado en el uso de prácticas de manejo en los ranchos ganaderos del país. Los pastizales y matorrales han sufrido disturbios, principalmente la apertura de tierras para cultivos y se encuentran deteriorados principalmente por el sobrepastoreo. La utilización de los pastizales y matorrales a través del pastoreo debería incluir una carga animal adecuada, sistemas de pastoreo y prácticas estratégicas de distribución del pastoreo. A pesar del deterioro, existe una gran diversidad de recursos genéticos, principalmente de pastos, que pueden ser utilizados para conservación y producción de semilla para rehabilitación de los pastizales. La resiembra, aunque de alto costo y riesgo, es una opción para rehabilitar aquellos pastizales o matorrales deteriorados. Estos ecosistemas pueden proporcionar servicios ambientales, principalmente captura de carbono para mitigar el cambio climático. Los retos son generar, transferir y aplicar conocimientos e innovaciones tecnológicas para lograr un manejo sostenible de los pastizales y matorrales, a pesar de algunas amenazas como la baja inversión en ciencia y tecnología, el cambio climático y la avaricia humana. Para lograr esto, es imprescindible la participación conjunta y comprometida de todos los actores e instituciones involucrados en el uso de estos ecosistemas.

Palabras clave: Condición pastizales, Manejo pastoreo, Recursos genéticos, Producción semilla, Resiembra pastizales, Carbono suelo.

Recibido: 23/11/2020

Aceptado: 26/05/2021

\section{Introducción}

Los pastizales y matorrales de las zonas árida y semiárida del centro y norte de México son recursos naturales que comprenden alrededor del $25 \%$ de la superficie nacional ${ }^{(1)}$ y tienen la capacidad de proporcionar varios productos y servicios ambientales a la sociedad. Uno de esos productos es la carne de rumiantes derivada de la ganadería extensiva, mientras que, de los servicios ambientales, la captura de carbono sería uno de los más importantes. Los pastizales y matorrales han sufrido grandes transformaciones debidas principalmente al cambio de uso de suelo, el sobrepastoreo y el clima, ocasionando un grave deterioro a estos recursos. 
La rehabilitación de los pastizales es una actividad necesaria para corregir su deterioro. El fuego es una alternativa ecológica y de bajo costo para el mejoramiento de pastizales ${ }^{(2,3)}$, mientras que el ajuste de carga animal en conjunto con prácticas para mejorar la distribución del pastoreo son recomendables para conservar o mejorar la condición de los pastizales áridos y semiáridos ${ }^{(4,5)}$. La resiembra de pastos, aunque es una práctica riesgosa y costosa, debido a las precipitaciones erráticas y el alto costo de preparación de terreno y adquisición de semilla, sigue siendo una opción para mejorar los pastizales.

En México, los estudios sobre manejo y rehabilitación de pastizales y matorrales se iniciaron en la década de los años 1950, en el Rancho Experimental La Campana del extinto Instituto Nacional de Investigaciones Pecuarias. Posteriormente, se intensificaron a partir de la fusión de los institutos de investigación agrícola, pecuaria y forestal y la creación del INIFAP en 1985, en sus Campos Experimentales Pecuarios del norte del país como "La Campana" en Chihuahua, "Carbó" en Sonora, "Aldama" en Tamaulipas y "Vaquerías" en Jalisco. Los resultados de estos trabajos están plasmados en un sinnúmero de publicaciones y han sido trasferidos a los productores a través de cursos, talleres y demostraciones. En el período 1970-1980, se contó con el apoyo de las organizaciones ganaderas, los gobiernos de los estados y el CONACYT, mientras que para los años de 1990 y principios de este siglo las Fundaciones Produce Estatales impulsaron la investigación y transferencia de tecnología en pastizales. En los últimos 20 años, los Programas Federales como el PROGAN de la SAGARPA, han apoyado al INIFAP para la capacitación de una gran cantidad de productores y técnicos en manejo de pastizales. Además, el INIFAP ha participado en la promoción del manejo de pastizales a través de la co-organización de Simposios, Congresos y Foros con diferentes Universidades del centro y norte de México, así como la Sociedad Mexicana de Manejo de Pastizales. El impacto socio-económico y ambiental de las investigaciones en pastizales realizadas en el INIFAP y otras instituciones es palpable en el manejo de los ranchos ganaderos. Por ejemplo, gran parte de los ranchos del norte y centro del país, incluyendo algunos ejidos, realizan prácticas de manejo del pastoreo, como el pastoreo diferido o descanso del pastizal, ajuste de carga animal y suplementación de ganado durante la sequía, lo que ha permitido la conservación y mejoramiento de la condición de algunas áreas de pastizales y matorrales. En la actualidad existe una gran cantidad de conocimientos e innovaciones tecnológicas disponibles para lograr un manejo sostenible de pastizales y matorrales, generados por el INIFAP, universidades y centros de investigación. En este documento, se presenta el estatus actual de los pastizales y matorrales áridos y semiáridos en algunos temas estratégicos, así como los principales retos y perspectivas a los que se tiene que enfrentar para conservar y aprovechar de forma sostenible dichos ecosistemas. 


\section{Condición de los pastizales y matorrales}

Los pastizales semiáridos de México se distribuyen en una franja de norte a sureste del país, desde Sonora hasta Guanajuato, mientras que los matorrales áridos se extienden ampliamente desde Baja California hasta Oaxaca ${ }^{(6)}$ (Figura 1). Estos ecosistemas han sufrido reducciones del $14 \%$ en pastizales y del $26 \%$ en matorrales por su conversión a la agricultura en los últimos 50 años, de tal manera que actualmente comprenden alrededor de 9.77 y 40.95 millones ha, respectivamente ${ }^{(1)}$. Desafortunadamente, el $95 \%$ de los pastizales y el $70 \%$ de los matorrales están sobre-pastoreados según fuentes oficiales (Figura 1).

Figura 1: Intensidad del pastoreo en pastizales y matorrales naturales de México. Fuente: Dirección General de Ordenamiento y Conservación de Ecosistemas, INE, SEMARNAT, México. 2003

Intensidad del pastoreo Sin sobrepastoreo

Matorrales de zonas áridas

Pastizal natural semidesértico

Con sobrepastoreo

Matorrales de zonas áridas

Pastizal natural semidesértico

No pecuaria

Matorrales de zonas áridas

Pastizal natural semidesértico

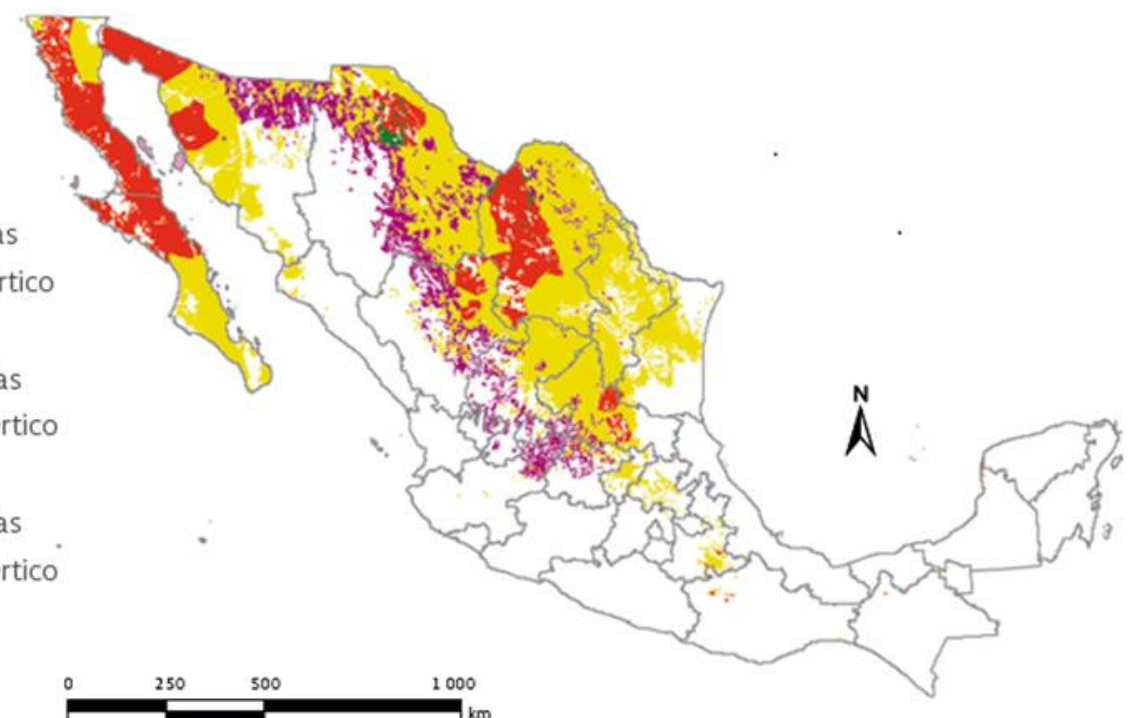

Los primeros estudios a nivel nacional durante las décadas de 1950 a 1970 indican que los pastizales semiáridos del centro y norte de México se constituían principalmente de gramíneas nativas de porte bajo y mediano como los pastos navajita (Bouteloua gracilis), banderita (B. curtipendula), navajita velluda (B. hirsuta), liebrero (B. rothrockii), navajita púrpura (B. radicosa), sabanilla (B. repens), navajita negra (B. eriopoda), navajita morada (B. chondrosioides), escorpión (B. scorpioides), tres barbas (Aristida adscencionis), tres barbas abierto (A. divaricata), tres barbas (A. ternipes), búfalo (B. dactyloides), mientras que en los pastizales áridos o halófitos los pastos más comunes incluían el toboso (Pleuraphis mutica), zacatón alcalino (Sporobolus airoides) y zacate salado (Distichlis spicata) ${ }^{(6)}$. Sin embargo, estudios recientes reportan algunos cambios en su composición florística y la aparición de gramíneas exóticas. En el noreste de Sonora, los pastizales semiáridos se 
componen principalmente de gramíneas nativas de los géneros Aristida, Bouteloua ${ }^{(7)}$, mientras que en las planicies centrales del estado, se localizan aproximadamente 800 mil hectáreas de zacate exótico buffel (Pennisetum ciliare) para uso ganadero y que fueron originalmente matorrales nativos ${ }^{(7)}$. Desgraciadamente, el sobrepastoreo ocasionado por una ganadería de carne en crecimiento, ha degradado el pastizal mediano y reducido su condición, afectando el ingreso económico de las familias ${ }^{(8)}$.

En el caso de los pastizales de Chihuahua, los pastos más comunes son de los géneros Bouteloua y Aristida y para los pastizales áridos, las especies dominantes son el toboso, zacatón alcalino y jigüite (Eragrostis obtusiflora) $)^{(9)}$. Además, se observa una invasión de arbustos como el garabatillo (Mimosa biuncifera) y varadulce (Aloysia gratissima), así como gramíneas exóticas como el africano (Eragrostis lemahnniana) y rosado (Melinis repens $)^{(9,10)}$. Estudios locales confirman un alto grado de deterioro, así como pérdidas desde 378 mil ha hasta 2.72 millones de hectáreas ${ }^{(11,12)}$, atribuidos a la apertura de tierras al cultivo, sobrepastoreo ${ }^{(10,12)}$, cambio climático, asentamientos humanos y políticas públicas inadecuadas en pastizales de Chihuahua ${ }^{(12)}$.

En Durango, los pastizales semiáridos se componen principalmente de gramíneas nativas de los géneros Aristida, Bouteloua, Elionurus, Eragrostis, Heteropogon, mientras que en los pastizales áridos, el zacate salado, zacate rasguño (Muhlenbergia asperifolia), zacatón alcalino, chino (Bouteloua ramosa), y toboso son los más comunes ${ }^{(13)}$. Sin embargo, la sobreexplotación de los pastizales, las condiciones climáticas adversas y la introducción e invasión de especies exóticas como el pasto rosado han ocasionado una reducción significativa de los pastizales del estado ${ }^{(13)}$, así como el cambio de una asociación de pastos Bouteloua-Bothriochloa a otra de rosado/barba negra (Heteropogon contortus) ${ }^{(14)}$.

Para los pastizales semiáridos de Zacatecas, las asociaciones más comunes son Aristida/Bothriochloa/Bouteloua y otros pastos exóticos como buffel, llorón (Eragrostis curvula), africano y rosado, mientras que en los pastizales áridos-halófitos el más común es el zacatón alcalino ${ }^{(15)}$. Además, los pastizales de Zacatecas mostraron que la mayoría se encuentran en estado de salud moderado, ya que aunque las propiedades de los suelos son aceptables, la vegetación presenta un alto grado de deterioro ${ }^{(16)}$.

En el caso de los pastizales semiáridos de Aguascalientes, los zacates dominantes son el navajita morada, navajita y lobero (Muhlenbergia phleoides), mostrando una buena condición con el $80 \%$ de vegetación original, sobre todo del género Bouteloua ${ }^{(17)}$. Para el estado de Jalisco, las comunidades principales son Bouteloua/Microchloa/Aristida en los pastizales semiáridos ${ }^{(18)}$ las cuales se encontraban en salud moderada a extrema en el año $2002^{(19)}$, siendo el principal problema el deterioro de la vegetación. En San Luis Potosí, las gramíneas más comunes son navajita, lobero, búfalo y los invasores buffel y rosado ${ }^{(20)}$. Desafortunadamente, un estudio reciente indica que el sobrepastoreo ha impactado la 
composición de los pastizales en el centro de México a través de la historia, pero que todavía es posible mejorar los pastizales ${ }^{(21)}$.

En la década de 1970, los matorrales del Desierto Sonorense estaban representados por arbustos como el saguaro (Carnegia gigantea), hierba del burro (Ambrosia dumosa), torote (Bursera microphylla), palo fierro (Olneya tesota), palo verde (Parkinsonia microphylla) y gobernadora (Larrea tridentata) ${ }^{(6)}$. Estudios recientes sobre la vegetación en Sonora, indican que los arbustos predominantes en la región de las planicies centrales son el palo fierro y rama blanca (Encelia farinosa), en la costa central el torote y Jatropha cinerea, en el altiplano el palo verde, Cylindropuntia, Opuntia y saguaro y en el Valle del Bajo Río Colorado la asociación Larrea/Ambrosia ${ }^{(7)}$. En el caso de los matorrales xerófilos del Desierto Chihuahuense estaban dominados por arbustos como la gobernadora, hojasén (Flourensia cernua), lechugilla (Agave lechuguilla), ocotillo (Fouquieria splendens), chaparro prieto (Acacia vernicosa) y otros de los géneros Opuntia, Dasylirion y Yucca ${ }^{(6)}$. En la actualidad, también son comunes la vara negra (Acacia neovernicosa), mezquite (Prosopis glandulosa), junco (Koeberlina spinosa), espino (Acacia berlandieri) y tecomblate (Condalia warnockii), mientras que en Coahuila se encuentra además Hechtia spp., candelilla (Euphorbia antisyphilitica), sangregado (Jatropha dioica) en el norte y Allenrolfea sp., Atriplex sp., Suaeda sp. y mezquite en el sur ${ }^{(22)}$. También se han reportado otros arbustos dominantes como el cenizo (Leucophyllum frutescens), mariola (Parthenium incanum), gobernadora, mezquite, lechuguilla, gatuño (Mimosa aculeaticarpa), espino y Gutierrezia microcephala en los matorrales xerófilos del norte de Coahuila ${ }^{(23)}$. Para la zona más árida del Desierto Chihuahuense en el sur de San Luis Potosí, se presentan arbustos como la gobernadora, hojasén, mezquite y chamizo (Atriplex canescens) ${ }^{(22)}$.

En Durango, los matorrales xerófilos están representados por gobernadora, hojasén, junco, mezquite, sangregado, candelilla, Opuntia así como asociaciones de lechuguilla/guapilla (Hechtia glomerata)/Ocotillo, siendo este último dominante en los matorrales del norte y centro del estado de Durango y sobresaliendo el género Henricksonia como endémico para los matorrales de Durango y Coahuila ${ }^{(24)}$. En contraste, los matorrales de Aguascalientes presentan diversas asociaciones donde las especies más comunes son nopales (Opuntia spp.), gatuño (Mimosa monancistra) y huizache (Acacia schaffneri) y gobernadora/mariola el cual presenta alta degradación, con sólo un $20 \%$ de su vegetación primaria ${ }^{(17)}$.

Para documentar los cambios de los pastizales y matorrales, debido al manejo y los efectos del clima, la disponibilidad de metodologías y herramientas prácticas es fundamental. El uso de sensores remotos ha mostrado ser una herramienta práctica para la estimación de forraje/productividad y cobertura vegetal ${ }^{(25,26)}$ y de la extensión y fragmentación de pastizales y matorrales ${ }^{(27)}$. 


\section{Manejo del pastoreo y utilización de pastizales y matorrales}

La utilización de los recursos forrajeros del pastizal como alimento primario representa la base del sistema de producción vaca-becerro, así como de la explotación de caprinos y ovinos bajo condiciones extensivas. El alarmante deterioro de los pastizales, ha sido atribuido en gran medida a la sobreutilización del recurso, por lo que el desarrollo de un programa adecuado de manejo del pastoreo debe considerarse como preventivo. Dicho programa se basa en una serie de principios ya establecidos y discutidos por numerosos autores ${ }^{(28,29)}$, destacando principalmente: a) la utilización de la carga animal óptima, b) la determinación de la mejor época de pastoreo, c) la implementación del sistema de pastoreo más adecuado, d) el uso de la especie o de la combinación de especies animales óptimas y e) el establecimiento de prácticas para una distribución uniforme del pastoreo.

Desde la década de 1980 se establecieron líneas de investigación tendientes a documentar los aspectos señalados. Los resultados de investigación obtenidos durante los primeros 50 años del Rancho Experimental La Campana, en la selección de la dieta, el consumo voluntario de forraje y la etología del ganado han sido detallados por algunos autores ${ }^{(30)}$. El Cuadro 1 muestra los rangos en algunos componentes de la dieta seleccionada por bovinos, con una marcada fluctuación estacional en dos tipos de pastizales y un matorral representativos del estado de Chihuahua ${ }^{(30)}$. Otros esfuerzos de investigación realizados en pastizales de Jalisco $^{(31,32)}$ y un matorral de Baja California Sur ${ }^{(33)}$ reportan tendencias similares que reflejan las deficiencias nutricionales de los pastizales y el matorral durante el período seco. De igual manera, en un estudio realizado en un pastizal mediano invadido de gatuño y huizache, la dieta de cabras presentó deficiencias de proteína durante la época de latencia de los pastos en Guanajuato $^{(34)}$.

Cuadro 1: Rangos reportados en componentes de la dieta de bovinos de carne en tres tipos de vegetación en el estado de Chihuahua

\begin{tabular}{llll}
\hline \multicolumn{1}{c}{ Componente } & $\begin{array}{l}\text { Pastizal } \\
\text { Mediano } \\
\text { abierto }\end{array}$ & $\begin{array}{l}\text { Pastizal } \\
\text { amacollado } \\
\text { abierto }\end{array}$ & $\begin{array}{l}\text { Matorral } \\
\text { inerme } \\
\text { parvifolio }\end{array}$ \\
\hline Proteína cruda, \% & $4.9-11.5$ & $5.3-11.8$ & $6.5-12.5$ \\
Energía metabolizable, Mcal kg ${ }^{-1}$ & $1.83-2.27$ & $1.7-2.38$ & $1.58-2.22$ \\
Digestibilidad, \% & $54.1-67.2$ & $50.6-70.6$ & $46.2-66.7$ \\
\hline \multicolumn{2}{l}{ Chávez y González ${ }^{(30)}$. }
\end{tabular}

En este orden de ideas, el consumo voluntario de forraje es sin duda el componente más importante en la regulación del balance energético del ganado en pastoreo. Asimismo, es una variable indispensable para una correcta estimación de la capacidad de carga de las tierras de pastoreo. Varias investigaciones ${ }^{(35,36)}$ han revisado ampliamente los factores que controlan el 
consumo voluntario de forraje, inherentes al animal y a las características de la vegetación, justificando la realización de estudios al respecto. Los valores de consumo promedio fluctúan entre el 1.8 a $3.5 \%$ del peso vivo, dependiendo del tipo de vegetación, la época del año y el estado fisiológico del ganado ${ }^{(30)}$. Datos cercanos fueron obtenidos en un pastizal amacollado arborescente de Chihuahua reportando consumos del 2.6 y $3.1 \%$ del peso vivo, para vacas gestantes y lactantes, respectivamente ${ }^{(37)}$.

De acuerdo a varios autores, la carga animal es el factor más crítico en la implementación de un esquema de manejo del pastoreo y utilización ${ }^{(4,38,39,40)}$. Los primeros estudios de utilización efectuados desde 1965 en el Rancho Experimental La Campana, mostraron un aumento de la cobertura vegetal utilizando cargas moderadas. Se ha evaluado la interacción entre la carga animal y los sistemas de pastoreo ${ }^{(5)}$, confirmando el impacto de la carga animal como factor primario de manejo.

En lo referente a la investigación sobre sistemas de pastoreo, algunos autores han señalado la complejidad estadística y los altos costos de este tipo de estudios ${ }^{(41)}$, lo que ha sido una limitante desde el punto de vista metodológico y financiero. De manera general, los sistemas de pastoreo rotacional han sido utilizados como una herramienta para contrarrestar los efectos no deseados del pastoreo selectivo ${ }^{(4)}$. Sin embargo, la evidencia generada durante los últimos 60 años, no indica una superioridad del pastoreo rotacional sobre el continuo, y las supuestas ventajas de los sistemas rotacionales, están fundadas más en la percepción de "interpretaciones anecdóticas" que en una evaluación objetiva de la evidencia experimental ${ }^{(39)}$. Finalmente, la investigación en sistemas de pastoreo no debe estar enfocada en encontrar el mejor método, sino en la identificar y cuantificar los principios y procesos del pastoreo que apoyen un manejo adaptativo y/o la toma de decisiones ${ }^{(42)}$.

En Jalisco, se encontró que el Pastoreo Rotacional Diferido mantiene la condición del pastizal, al compararlo con el pastoreo continuo ${ }^{(43)}$. Los sistemas de pastoreo de corta duración (PCD), también han sido materia de evaluación, con resultados no muy favorables. Por ejemplo, este sistema mostró una reducción del $49 \%$ en la cobertura vegetal en un pastizal de Chihuahua ${ }^{(44)}$. Esa misma tendencia negativa del PCD se observó después de 12 años de evaluación en tres pastizales y un matorral de Chihuahua ${ }^{(45)}$, con decremento del $75 \%$ en la cobertura vegetal, mientras que en el pastoreo continuo (PCT), se obtuvo un incremento del $124 \%$. Resultados similares han sido reportados en un estudio de siete años en un pastizal semiárido de Jalisco ${ }^{(46)}$, con reducciones en la producción de biomasa, del orden de 72 y $43 \%$ para el PCD y PCT, respectivamente. Además, se ha observado un incremento de la disponibilidad de forraje a mayor distancia del agua, llegando hasta $110 \%$ a $1 \mathrm{~km}$ del centro de la célula de pastoreo, sugiriendo una distribución poco eficiente del pastoreo con PCD en un pastizal de Chihuahua ${ }^{(44)}$. 


\section{Recursos genéticos y variedades de pastos}

El deterioro de los pastizales ha provocado la necesidad de conservar germoplasma o diversidad de recursos genéticos forrajeros para buscar entre ellos alternativas vegetales que estabilicen tanto los ecosistemas como la economía de los pastizales en México ${ }^{(47)}$. Para ello, el INIFAP, comenzó a colectar y evaluar germoplasma de gramíneas nativas e introducidas desde finales de 1970, en colaboración con bancos de germoplasma de diferentes partes del mundo. Algunas de las especies nativas de mayor interés han sido el banderita y navajita, y especies introducidas como el llorón, garrapata (Eragrostis superba) y buffel. El germoplasma se estableció en diferentes estados de México, logrando mayor persistencia las evaluaciones en San Luis Potosí y como resultado se identificaron accesiones sobresalientes, para posteriormente registrarse ante el Servicio Nacional de Inspección y Certificación de Semillas (SNICS). En pasto banderilla, se han evaluado accesiones provenientes de diferentes estados de la república, identificándose varios ecotipos sobresalientes para producción de forraje como el INIA-235-ZAC, INIA-426-COAH, INIA-315-JAL, con valores de 3.0 a $3.1 \mathrm{t}$ de materia seca $(\mathrm{MS}) \mathrm{ha}^{-1}$, de un total de 59 accesiones $^{(48)}$, mientras que, en San Luis Potosí se evaluaron 147 accesiones de varios estados del centro del país y como resultado, se obtuvo la variedad denominada "Banderilla Diana", con rendimientos medios en condiciones de temporal de $1.85 \mathrm{t} \mathrm{MS} \mathrm{ha}^{-1}$, con proteína cruda (PC) de $8.6 \%$ en la etapa de floración y $3.6 \%$ en madurez ${ }^{(49)}$. En pasto navajita, la variedad "Cecilia" se obtuvo de un programa de selección, donde se evaluaron 53 genotipos de varios estados del centro del país. Esta variedad, puede producir hasta $0.98 \mathrm{t} \mathrm{MS} \mathrm{ha}^{-1}$, con $9.7 \%$ de PC en la etapa de floración y $3.4 \%$ en madurez ${ }^{(50)}$. Respecto a las especies introducidas, se seleccionó y registró la variedad Llorón "Imperial", de 205 genotipos evaluados. Esta variedad puede obtener un rendimiento medio de $1.2 \mathrm{t} \mathrm{MS} \mathrm{ha}^{-1}$, en condiciones de temporal. Además, presenta un contenido de PC en inicio de floración de $10.2 \%$ y de $4.6 \%$ en madurez ${ }^{(51)}$. En la colección de pasto garrapata, la accesión sobresaliente de 14 genotipos fue "Hércules" cuyo rendimiento promedio en condiciones de temporal fue $1.2 \mathrm{t} \mathrm{MS} \mathrm{ha}{ }^{-1}, \mathrm{PC}$ de $10.2 \%$ en inicio de floración y $4.6 \%$ en madurez ${ }^{(52)}$. En el germoplasma de pasto buffel, de las 78 accesiones evaluadas, se identificaron dos genotipos sobresalientes y se registraron como "Titan" y "Regio". Los rendimientos registrados para estas variedades fueron 2.12 y $2.25 \mathrm{t}$ MS ha ${ }^{-1}$, con PC en floración de 6.1 y $5.8 \%$, mientras que en madurez $4.0 \%$ y $3.3 \%$, respectivamente ${ }^{(53)}$. En el noreste de México, se encuentran disponibles variedades del pasto buffel, "Milenio" para los estados de Tamaulipas y Nuevo León ${ }^{(54)}$ y "Zaragoza 115" y "Zaragoza 119" para el estado de Coahuila ${ }^{(55)}$.

En los últimos años, se han realizado recolecciones de material vegetativo y se han evaluado atributos morfológicos, productivos y genéticos de pastos nativos. Por ejemplo, investigadores del Colegio de Postgraduados lograron identificar y registrar las variedades NDEM-5, NDEM-125, NDEM-303, NDEM-417 y NDEM-LA ZARCA de pasto 
banderita $^{(56)}$. En México se ha reportado una amplia diversidad genética, morfológica y productiva de este pasto, con rendimientos desde 13.7 g hasta 1,213 $\mathrm{g} \mathrm{MS} \mathrm{planta}^{-1}$ de una colecta realizada en 13 estados, desde Sonora hasta Guerrero ${ }^{(56,57)}$. Además, en una colecta de 55 poblaciones de banderita del estado de Chihuahua se encontraron valores desde 4 a 260 g MS planta ${ }^{-1(58)}$. En un banco de germoplasma establecido en Zacatecas con 17 ecotipos, se encontraron rendimientos durante el periodo de lluvias desde 1,320 hasta 3,337 $\mathrm{kg}$ MS $\mathrm{ha}^{-1(59)}$. La diversidad morfológica dentro de esta especie se puede atribuir a su gran variación en el nivel de ploidía ${ }^{(60)}$, ya que otras investigaciones mencionan que el nivel de ploidía puede tener efectos en la anatomía, morfología y fisiología de las plantas ${ }^{(61)}$.

Respecto al pasto navajita, se ha encontrado una gran diversidad genética dentro de esta especie $^{(62)}$, lo cual puede deberse a que el centro de origen de esta especie se encuentra en el centro de México ${ }^{(63)}$. Así mismo, existe la posibilidad de seleccionar germoplasma de pasto navajita de alta producción, ya que de sólo 145 ecotipos colectados en Chihuahua, se reportó un rango de rendimiento desde 0.3 hasta $48 \mathrm{~g} \mathrm{MS} \mathrm{planta}^{-1}$ en un solo corte ${ }^{(64)}$. Mientras que en Zacatecas se observaron rendimientos desde 842 a 1,957 kg MS ha- ${ }^{-1}$ en 20 genotipos $^{(59)}$. En zacate punta blanca (Digitaria californica) se ha encontrado gran diversidad genética y morfológica en Chihuahua, reportando dos accesiones sobresalientes en la producción de forraje, de 91 ecotipos colectados ${ }^{(65)}$. Para pasto gigante (Leptochloa dubia) se encontró una amplia variabilidad genética en 32 genotipos, con rendimientos desde 6 hasta 174 g MS planta ${ }^{-1(66)}$. Por último, en zacate tempranero (Setaria macrostachya) se concluyó que existe una amplia variación genética y se lograron identificar tres poblaciones con potencial forrajero, de una colección realizada en $44 \operatorname{sitios}^{(67)}$.

\section{Producción de semilla de pastos}

El principal valor de la producción de semillas de zacates es para la resiembra o rehabilitación de tierras de pastoreo, pero también tiene un valor importantísimo para la conservación de suelos. Sin embargo, la producción comercial de semilla de zacates para zonas semiáridas tiene un reciente desarrollo en México. Del año 2001 al 2016 en el Catálogo Nacional de Variedades Vegetales ${ }^{(68)}$, se tiene documentado el registro definitivo de 27 variedades e híbridos de zacates, de los cuales, 12 son aptos para las zonas semiáridas y pertenecen a cinco especies, por lo tanto, se puede decir que ya se cuenta con material genético para producir semilla calificada.

Los trabajos de investigación sobre producción de semilla de zacates para zonas áridas y semiáridas, datan de la década de los años 1980 y se pueden observar en el Cuadro 2. En dichos trabajos, se ha determinado principalmente el volumen de producción y características de calidad de la semilla, en algunos casos con ensayos de fertilización y riego. Las dos especies más estudiadas han sido los pastos buffel y banderita. 
Cuadro 2: Producción máxima de semilla de diferentes especies de pastos en las zonas áridas y semiáridas de México

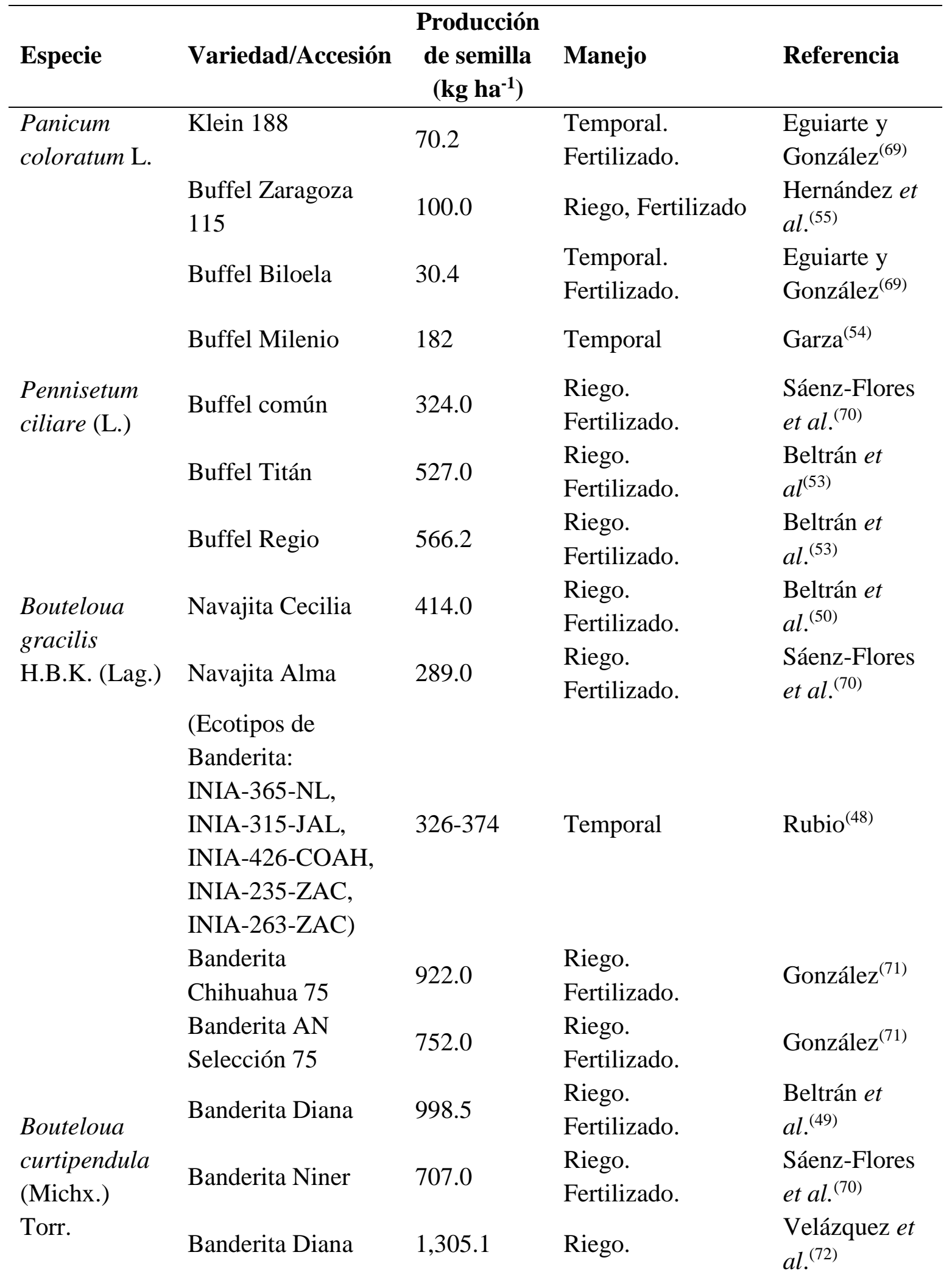




\begin{tabular}{|c|c|c|c|c|}
\hline & & & $\begin{array}{l}\text { Fertilizado. Una } \\
\text { cosecha, segundo } \\
\text { año de establecido. }\end{array}$ & \\
\hline $\begin{array}{l}\text { Leptochloa } \\
\text { dubia (Kunth) } \\
\text { Nees. }\end{array}$ & Gigante Van Horn & 670.0 & $\begin{array}{l}\text { Con riego. } \\
\text { Fertilizado. }\end{array}$ & $\begin{array}{l}\text { Sáenz-Flores } \\
\text { et al. }{ }^{(70)}\end{array}$ \\
\hline $\begin{array}{l}\text { Eragrostis } \\
\text { curvula } \\
\text { (Schrad) Nees }\end{array}$ & Llorón Imperial & 685.0 & $\begin{array}{l}\text { Con riego. } \\
\text { Fertilizado. Una } \\
\text { cosecha en un año. }\end{array}$ & $\begin{array}{l}\text { Beltrán et } \\
a l . .^{(51)}\end{array}$ \\
\hline Eragrostis & Garrapata & 1619.0 & $\begin{array}{l}\text { Con riego. } \\
\text { Fertilizado. }\end{array}$ & $\begin{array}{l}\text { Sáenz-Flores } \\
\text { et al. }{ }^{(70)}\end{array}$ \\
\hline $\begin{array}{l}\text { superba } \\
\text { (Peyr). }\end{array}$ & Garrapata Hércules & 1651.0 & $\begin{array}{l}\text { Con riego. } \\
\text { Fertilizado. Una } \\
\text { cosecha en un año. }\end{array}$ & $\begin{array}{l}\text { Beltrán et } \\
a l .{ }^{(52)}\end{array}$ \\
\hline
\end{tabular}

\section{Resiembra de pastizales en zonas áridas y semiáridas}

Considerando el deterioro de los pastizales y matorrales, la resiembra de pastos es una práctica común para revertir dicho deterioro que consiste en establecer vegetación a través de la diseminación artificial de semilla de una sola especie o en mezcla, utilizando especies adaptadas y camas de siembra apropiadas. Esto con el fin de aumentar la productividad y calidad forrajera y carga animal y diversificación de dieta para el ganado, además, de reducir la erosión e invasión de especies menos deseadas; sin embargo, esta práctica es costosa y de alto riesgo. En general, se recomienda la resiembra de pastos en sitios que presenten áreas con menos del $15 \%$ de cobertura de pastos nativos ${ }^{(73,74,75)}$.

Es muy importante utilizar especies adaptadas a las condiciones de suelo y clima del área a resembrar. Las especies nativas se adaptan mejor a las diferentes condiciones de clima, suelo y son más persistentes; pero son más difíciles de establecer. Por lo cual, se sugiere que el origen de las semillas no sea de más de $300 \mathrm{~km}$ del sitio donde se va a resembrar. Por lo general, se recomienda utilizar una mezcla de especies, de preferencia nativas. La ventaja de la mezcla radica en que la diversidad de especies puede hacer un mejor aprovechamiento de la variabilidad en las condiciones del suelo de los agostaderos ${ }^{(76)}$. Los principales pastos forrajeros utilizados para la siembra o resiembra son: banderita y navajita de los nativos y llorón, garrapata y buffel de los introducidos ${ }^{(77)}$.

La presencia o falta de humedad en el suelo, es la variable más importante para el establecimiento de las plántulas de los zacates. Por lo tanto, se debe de preparar el terreno para la siembra, lo que se conoce como "cama de siembra"; ésta tiene como función aflojar 
el suelo, darle mayor porosidad, para que retenga mayor cantidad de agua y proporcionar condiciones más favorables para el establecimiento de los zacates en estos ambientes ${ }^{(76,77)}$.

Existen muchas prácticas para resiembra de pastizales, que van desde subsoleo, barbecho, rastreo, bordos a nivel, surcados "lister" y el uso de rodillo aireador entre otras opciones. Este último, realiza uno de los procesos más rápidos y eficaces, sobre todo en matorrales desérticos, ya que aumenta la capacidad de infiltración de agua, disminuye la compactación del suelo ${ }^{(78)}$ y ha permitido un buen establecimiento de pastos como navajita, banderita, punta blanca y buffel ${ }^{(76,79,80)}$ en Sonora, Chihuahua y Coahuila. Sin embargo, prácticas como el subsoleo más barbecho y rastreo también favorecen la cobertura con valores hasta $80 \%$ y un rendimiento de 5.0 a $13.6 \mathrm{t} \mathrm{MS} \mathrm{ha}^{-1}$ en zacate buffel para el estado de Coahuila y Jalisco, respectivamente ${ }^{(81,82)}$. Para el estado de Sonora, el desmonte total ha tenido repercusiones en pérdida de suelo y diversidad vegetal, por lo tanto, debe evitarse en la preparación de la cama de siembra ${ }^{(83)}$. A raíz de que existen diferentes resultados con métodos de siembra, se considera necesario enfatizar que las prácticas deberán ajustarse a condiciones ambientales particulares. Por otra parte, es recomendable mover suelos cuando haya algo de humedad y dar uno o dos pasos de rastra para hacer una buena cama de siembra ${ }^{(84)}$. Sin embargo, es preferible sembrar en húmedo al inicio de la época de lluvias para incrementar la oportunidad de germinación y establecimiento de plantas. Se puede preparar el terreno con anticipación, ya sea "rayado" con arado a contrapendiente o barbecho y paso de rastra. Las siembras en seco son más riesgosas, por lo cual sería conveniente realizar la siembra una o dos semanas antes de la época de lluvias para evitar pérdida de semilla ${ }^{(77)}$.

Posterior a la siembra, durante el primer año las malezas pueden competir por luz, espacio y nutrientes del suelo, lo que disminuirá el potencial de los pastos. Se recomienda controlar la maleza principalmente con herbicida selectivo. Del segundo año en adelante, para mantener en buen estado la producción de forraje, se recomienda al inicio de la época de lluvias realizar labores de control de maleza química o manual, aunque es una práctica costosa ${ }^{(77)}$. Sin embargo, esta práctica sí es recomendable para control de arbustos, cuando se incrementa su densidad.

La fertilización estará en función de la cantidad de precipitación, en años muy secos con precipitación menor a $200 \mathrm{~mm}$ no se recomienda fertilizar. En el primer año la fertilización se realiza con la fórmula 20-20-00 y sólo cuando la condición de humedad sea favorable. Una vez establecido el pasto se sugiere fertilizar cada año ${ }^{(77)}$. Sin embargo, los fertilizantes deben aplicarse con reserva debido a los altos costos y a la variabilidad temporal y espacial de las precipitaciones. Otras opciones con buenos resultados en resiembras de pastos nativos, son la aplicación de biofertilizantes ${ }^{(85)}$ y subproductos orgánicos como los biosólidos ${ }^{(86)}$. 


\section{Almacén y captura de carbono en pastizales y matorrales}

Los pastizales y matorrales pueden, al igual que los bosques, proporcionar varios servicios ambientales para la sociedad, entre ellos la captura de carbono. Actualmente, los pastizales del mundo tienen buen potencial para la mitigación del cambio climático a través de la captura de carbono ${ }^{(87,88)}$ que podría alcanzar hasta $148 \mathrm{Tg} \mathrm{CO}_{2}$ año $^{-1}$ a través de la implementación de estrategias de manejo del pastoreo ${ }^{(88)}$.

En México, los pastizales con pastoreo moderado presentan mayor almacén de carbono del suelo ( $800 \mathrm{~g} \mathrm{C} \mathrm{m}^{-2}$ ) comparado con pastizales que han sido sobrepastoreados $\left(650 \mathrm{~g} \mathrm{C}^{2}\right.$ $\left.\mathrm{m}^{-2}\right)^{(89)}$. Otro estudio ${ }^{(90)}$ encontró resultados similares, con mayores almacenes de carbono en suelos de pastizales con pastoreo moderado y alta cobertura de pastos forrajeros en comparación con pastizales sobrepastoreados y con baja cobertura de plantas, con un promedio de 34.5 y $24.3 \mathrm{t} \mathrm{Cha}^{-1}$ a $0.3 \mathrm{~m}$ de profundidad de suelo en pastizales semiáridos y halófitos, respectivamente. Considerando una capacidad de captura de carbono en suelo de $0.1 \mathrm{tC} \mathrm{ha}^{-1} \mathrm{año}^{-1(91)}$ y una superficie de 9.77 millones ha, los pastizales semiáridos de México podrían capturar aproximadamente $3.5 \mathrm{Tg} \mathrm{CO}_{2}$ año $^{-1}$, con un manejo apropiado del pastoreo. Respecto a los matorrales, no se encontraron diferencias muy marcadas con el pastoreo, aunque los almacenes de carbono del suelo son bajos con 21.7 y $23.0 \mathrm{t} \mathrm{C} \mathrm{ha}^{-1}$ a $0.3 \mathrm{~m}$ de profundidad en los matorrales de gobernadora y lechuguilla, respectivamente, en el Desierto Chihuahuense ${ }^{(90)}$. Las zonas semiáridas almacenan más carbono que las zonas áridas de México y en el caso de los pastizales el suelo almacena el $90 \%$ del carbono, mientras que el suelo de los matorrales almacena solamente el $45 \%$ del carbono ${ }^{(92)}$.

En relación a la captura de carbono, se ha reportado que los pastizales nativos o naturales son sumideros de carbono, ya que pueden capturar hasta $0.054 \mathrm{t} \mathrm{CO}_{2}$ ha $^{-1}$ día $^{-1(93)}$. Otros autores ${ }^{(94)}$ concluyen que el potencial de captura de carbono de la biomasa en pastizales inducidos varía de 0.99 a 1.51 t CO$_{2} \mathrm{eq} \mathrm{ha}^{-1}$ año $^{-1}$ después de 30 y 10 años de abandono, respectivamente. Contrario a lo esperado, un pastizal semiárido con alta cobertura de plantas mostró ser neutral como fuente de carbono, y el pastizal con baja cobertura de plantas mostró ser un sumidero de carbono, mientras que la composición de especies no influyó en el balance de carbono ${ }^{(95)}$. Además, en otro estudio se concluye que los pastizales semiáridos podrían pasar de sumideros de carbono a fuentes de carbono por efectos del cambio climático ${ }^{(96)}$.

El uso de imágenes digitales y modelos de regresión, aunque presentaron bajo ajuste, tienen potencial para la predicción de carbono del suelo ${ }^{(97)}$. Otros autores proponen un modelo ecológico para la estimación de carbono del suelo en pastizales semiáridos, con variables como la cobertura de pastos forrajeros, la precipitación media anual y el contenido de arena del suelo ${ }^{(98)}$. 


\section{Retos y perspectivas}

Dada la gran disminución de las superficies de los pastizales y matorrales, así como su alto deterioro, es apremiante detener el avance de la frontera agrícola y evitar el sobrepastoreo. Para esto, es urgente el cumplimiento de leyes federales y estatales vigentes sobre conservación y uso sostenible de ecosistemas, así como un programa de transferencia de tecnología rural con el fin de capacitar y concientizar a todos los ganaderos sobre el manejo sostenible de pastizales y matorrales en la región centro-norte del país. En la actualidad se dispone de tecnologías como el uso de imágenes y los sistemas de información geográfica para el monitoreo y evaluación de pastizales y matorrales. Sin embargo, es importante continuar con el desarrollo de tecnologías que permitan la detección más fina de los procesos y la estructura de los pastizales, como la identificación de plantas a nivel del suelo, con el fin de facilitar la toma de decisiones en pequeña y gran escala.

Respecto a la utilización de pastizales, es urgente implementar estrategias de manejo del pastoreo que se adapten a las condiciones de la vegetación y el clima de cada rancho, pero, sobre todo, un ajuste de carga animal que permita un uso moderado de los pastizales. También es necesario reactivar los estudios de calidad de la dieta, selectividad, consumo de forraje y estrategias de manejo del pastoreo para lograr un uso eficiente de los recursos y la respuesta de la vegetación, en un enfoque más ecosistémico, y con la finalidad de maximizar la producción animal y conservar el pastizal.

En relación a la conservación de recursos genéticos, se han evaluado más de 1,200 ecotipos de pastos nativos e introducidos ${ }^{(48-53,56,57,58)}$. No obstante, a la fecha solamente se han generado ocho variedades de pasto banderita, ocho de pasto buffel y una de cada uno de los pastos navajita, llorón y garrapata ${ }^{(99)}$. Por esta razón, es importante diseñar estrategias de financiamiento que permitan continuar con estudios de diversidad y programas de mejoramiento genético. Además, es necesario incursionar en la búsqueda de otras especies forrajeras que habitan en el árido y semiárido de México, ya que se ha reportado que existen más de 300 especies de gramíneas en estas regiones ${ }^{(13)}$. Estas acciones permitirían generar nuevas variedades de pastos, con buen potencial de establecimiento y producción de forraje ante los retos del cambio climático.

Actualmente, se puede producir semilla de pastos nativos con las tecnologías disponibles a un costo menor que la semilla importada. Aunque los retos principales son bajar los costos de producción, e incrementar el rendimiento y calidad de semilla. En el futuro inmediato se debe investigar más sobre el vigor de la semilla, escarificación y recubrimiento y fijación de productos como nutrientes o insecticidas en la semilla, para mejorar su desempeño en el campo. Posteriormente, es fundamental la generación de paquetes tecnológicos para la producción de semilla de diferentes pastos, evaluando el uso de prácticas agronómicas y mecanización de los procesos de cosecha y beneficio de semilla. 
Para la resiembra de pastos, se recomienda revegetar aquellos pastizales degradados, de preferencia con plantas nativas, con el fin de recuperar su estructura y funcionamiento para la producción de bienes y servicios para la sociedad. En esta actividad, la disponibilidad de semillas de especies nativas es un gran reto para solventar, pues, aunque existen variedades liberadas por INIFAP y otras instituciones, como navajita y banderita, la cantidad disponible es insuficiente para atender la demanda. El establecimiento de semilleros con productores cooperantes es una opción viable. En segundo término, es urgente el diseño de equipo simple, práctico y de bajo costo para la preparación de terreno para la resiembra de pastos.

Sobre la captura de carbono en pastizales y matorrales, es apremiante la implementación del pastoreo moderado en ejidos y ranchos ganaderos para disminuir las emisiones de carbono e incrementar la captura de este mismo gas. Algunos retos importantes son generar metodologías confiables, rápidas y sencillas para la estimación de almacenes y captura de carbono, incluir el manejo sostenible de pastizales y matorrales como una opción para los compradores de créditos de carbono en los mercados oficiales o voluntarios, y promover las ventajas del manejo del pastoreo para incrementar la captura de carbono a través de una ganadería sostenible.

El cambio climático ya está afectando los pastizales y disminuirá en forma directa y mucho más dramática la productividad y el aporte de servicios ambientales de los pastizales y matorrales del norte de México. Esto impactará la capacidad de carga de los pastizales con reducciones en la producción de carne, pérdida de biodiversidad, disminución en la capacidad de captura de carbono y afectaciones en el ciclo hidrológico de estos ecosistemas. Las nuevas condiciones climáticas obligan a realizar investigaciones integrales para encontrar las alternativas de manejo y rehabilitación de estos ecosistemas acorde a dichos cambios.

\section{Literatura citada:}

1. SEMARNAT. Secretaría del Medio Ambiente y Recursos Naturales. Ecosistemas terrestres. En: Informe de la situación del medio ambiente en México 2015. México. 2016.

https://apps1.semarnat.gob.mx:8443/dgeia/informe15/tema/pdf/Cap2_Ecosistemas.pdf. Consultado 17 Sep, 2020.

2. Jurado GP, Negrete RLF, Chávez RMG. Efecto del fuego sobre el control de escobilla (Haplopappus venetus) y la productividad de un pastizal en Jalisco. Manejo Pastiz 1990;3(3):33-36.

3. Luna M, Britton CM, Rideout-Hanzak S, Villalobos C, Sosebee RE, Wester DB. Season and intensity of burning on two grass species of the Chihuahuan desert. Range Ecol Manage 2014;67(6):614-620. 
4. Bailey DD, Brown JR. Rotational grazing systems and livestock grazing behavior in shrub-dominates semiarid and arid rangelands. Range Ecol Manage 2011;64(1):1-9.

5. Chávez AH, Pérez A, Sánchez E. Intensidades de pastoreo y esquemas de utilización en la selección de la dieta del ganado durante la sequía. Tec Pecu Mex 2000;38(1):19-34.

6. Rzedowski J. Vegetación de México. 1ra ed. digital. México: Comisión Nacional para el Conocimiento y Uso de la Biodiversidad; 2006.

7. Martínez-Yrízar A, Felger SR, Búrquez A. Los ecosistemas terrestres de Sonora: un diverso capital natural. En: Molina FF, Van-Devender T editores. Diversidad biológica del estado de Sonora. México. 2009:129-156.

8. Ibarra-Flores F, Martin M, Medina S, Ibarra F, Retes R. Cambios de vegetación y costos asociados con el continuo sobrepastoreo del ganado en el pastizal mediano abierto de Cananea, Sonora, México. Rev Mex Agron 2018;42(1):855-866.

9. Royo M. Descripción geográfica y fisiográfica del pastizal. En: La biodiversidad en Chihuahua: estudio de estado. México. CONABIO; 2014:262-267.

10. Valerio A, Carreón E, Lafón A, Ochoa JM, Calderón P, Soto DM. Distribución, extensión espacial y condición de los pastizales en el estado de Chihuahua. Chihuahua, México. Profauna-TNC; 2005.

11. Royo M, Sierra JS, Morales NC, Melgoza A, Jurado P. Estudios ecológicos de pastizales. En: Chávez A, Carrillo R compiladores. Rancho Experimental La Campana 50 Años de Investigación y Transferencia de Tecnología en Pastizales y Producción Animal. 1ra ed. Chihuahua, Chih., México: SAGARPA-INIFAP-Sitio Experimental La Campana-Madera; 2008:23-70.

12. De la Maza BM, Banda I, Mendoza G, Leal OA, Rendón G. Reporte del estado de los pastizales del Desierto Chihuahuense. Pronatura Noreste-American Bird Conservancy; 2019.

13. Herrera AY, Cortés OA. Diversidad de las gramíneas de Durango, México. Polibotánica 2009;(28):49-68.

14. Herrera CJ, Herrera AY, Carrete CFO, Almaraz AN, Naranjo JN, González GF. Cambio en la población de gramíneas en un pastizal abierto bajo sistema de pastoreo continuo en el norte de México. Interciencia 2011;36(4):300-305.

15. Herrera Y, Cortés A. Diversidad y distribución de las gramíneas (Poaceae) en el estado de Zacatecas. J Bot Res Inst Texas 2009;3(21):775-792. 
16. Echavarría-Cháirez F, Santos JL, Gutiérrez R, Medina G. Validación de una estrategia metodológica para la evaluación cualitativa de un pastizal mediano abierto del estado de Zacatecas. Rev Mex Cienc Pecu 2015;6(2):171-191.

17. Siqueiros-Delgado MA, Rodríguez AJA, Martínez RJ, Sierra MJC. Situación actual de la vegetación del estado de Aguascalientes, México. Bot Sci 2016;94(3):455-470.

18. Aguado SGA, García ME, Velasco GC, Flores FJL. Importancia de los elementos climáticos en la variación florística temporal de pastizales semidesérticos. Acta Bot Mex 1996;35:65-81.

19. Jurado P, Luna M, Barretero R, Royo M. Rangeland health of semiarid grasslands in Jalisco. In: Aguirre-Bravo C, et al. editors. Monitoring science and technology symposium: unifying knowledge for sustainability in the western hemisphere. Fort Collins, Col. USA; 2006.

20. Retana RFI. Distribución y riqueza de las gramíneas de San Luis Potosí [tesis maestría]. Durango, Dgo.: Instituto Politécnico Nacional; 2017.

21. Mellink E, Riojas ME. Livestock and grassland interrelationship along five centuries of ranching the semiarid grasslands on the southern highlands of the Mexican Plateau. Elem Sci Anthrop 2020;8:20. DOI: https://doi.org/10.1525/elementa.416.

22. Granados-Sánchez D, Sánchez A, Granados VRL, Borja DA. Ecología de la vegetación del Desierto Chihuahuense. Rev Chapingo Serie Cienc Forest Amb 2011;17:111-130.

23. Encina-Domínguez JA, Arévalo JR, Villareal QJA, Estrada CE. Composición, estructura y riqueza de plantas vasculares del matorral xerófilo en el norte de Coahuila. Bot Sci 2020;98(1):1-15.

24. González EMS, González EM, Márquez LMA. Vegetación y ecorregiones de Durango. CIIDIR Durango-IPN-Gobierno del estado de Durango. Durango, Dgo. 2006.

25. Medina G, Gutiérrez R, Echavarría F, Amador M, Corral JA. Estimación de producción de forraje con imágenes de satélite en pastizales de Zacatecas. Rev Mex Cienc Pecu 2009;47(2):135-144.

26. Chávez CE, Paz PF, Bolaños GMA. Estimación de biomasa y cobertura aérea usando radiometría e imágenes digitales a nivel de campo en pastizales y matorrales. Terra Latinoam 2017;35:247-257.

27. De León GD, Pinedo A, Martínez JH. Aplicación de sensores remotos en el análisis de la fragmentación del paisaje en Cuchillas de la Zarca, México. Investig Geogr 2014;84:42-53. 
28. Vallentine JF. Grazing management. San Diego, USA: Academic Press Inc.; 1990.

29. Chávez RMG, Soltero S. Manejo del pastoreo y utilización del recurso pastizal. En: Aguado A, Chávez G editores. Guía para el manejo de ranchos ganaderos del Altiplano Central. Ojuelos, Jalisco, México: SARH-INIFAP; 1993:10-21.

30. Chávez SA, González GF. Estudios zootécnicos I. Animales en pastoreo. En: A. Chávez A, Carrillo R editores. Rancho Experimental La Campana 50 años de investigación y transferencia de tecnología en pastizales y producción animal. Chihuahua, Chih. México: INIFAP-CIRNOC-Sitio Experimental La Campana-Madera; 2008:113-183.

31. Chávez G, Luna M, Jurado P, Aguado A. Valor nutricional de la dieta de bovinos en un pastizal mediano de los Altos de Jalisco. Manejo Pastiz 1988;1(1):33-36.

32. Chávez RMG, Luna LM. Comparación del valor nutritivo de la dieta seleccionada por bovinos en un sistema de apacentamiento de corta duración vs uso continuo. Manejo Pastiz 1991;4(3):14-19.

33. Chávez RG, Avalos R. Diet selection of bovine in arbocrasicaulescent shrubland associated with buffelgrass (Cenchrus ciliaris) in northwest of Mexico. In: Proc $24^{\text {th }}$ World Buiatrics Congress. Nice, France. 2006.

34. Barbosa JER, Córdova DG. Valor nutritivo de la dieta seleccionada por cabras en un pastizal con alta densidad de gatuño (Mimosa biuncifera). Manejo Pastiz 1992;5(3):110114.

35. Chávez RMG. Consumo voluntario de forraje de rumiantes en libre pastoreo. En: CursoTaller Internacional sobre Consumo Voluntario de Alimento. UAAAN-GNMNA. Saltillo, Coahuila. 1995:70-85.

36. Mejía HJ. Consumo voluntario de forraje por rumiantes en pastoreo. Acta Universitaria UG 2002;12(3);56-63.

37. Chávez RMG. Consumo voluntario de forraje, valor nutritivo de la dieta y gasto energético de vacas gestantes y lactantes en pastoreo [tesis maestría]. Universidad Autónoma de Chihuahua; 1990.

38. Derner JD, Hart R, Smith MA, Waggoner JW. Long-term cattle gain responses to stocking rate and grazing systems in northern mixed-grass prairie. Livestock Sci 2008;117:60-69.

39. Briske DD, Derner JD, Brown JR, Fuhlendorf SD, Teague WR, Havstad KM, et al. Rotational grazing on rangelands: Reconciliation of perception and experimental evidence. Range Ecol Manage 2008;61(1):3-17. 
40. Distel R. Manejo del pastoreo en pastizales de zonas áridas y semiáridas. Rev Argentina Prod Anim 2013;33(1):53-64.

41. Reuter RR, Moffet CA. Invited Review: Designing a grazing experiment that can reliably detect meaningful differences. The Professional Animal Scientist 2016;32:1930; http://dx.doi.org/10.15232/pas.2015-01424.

42. Kothmann MM. Grazing methods: A view point. Rangelands 2009;31:5-10.

43. Giner RA, Negrete LF, Luna LM. Efecto del pastoreo rotacional diferido sobre un pastizal mediano abierto del NE de Jalisco. En: II Congreso Nacional de Manejo de Pastizales-UAAAN. Saltillo, Coah. 1986:37.

44. Soltero S, Bryant F, Melgoza A. Standing crop patterns under short duration grazing in northern Mexico. J Range Manage 1989;42(1):20-21.

45. Gonzalez GFJ. Vegetation changes after 12 years in four private ranches under short duration and continuous grazing systems in Chihuahua, Mexico [doctoral thesis]. Lubbock, Texas USA: Texas Tech University; 2006.

46. Luna LM, Chávez RMG. Sistemas de pastoreo. En: IV Reunión Anual de Capacitación COTECOCA-SARH. Guadalajara, Jal. 1992:17-22.

47. Arredondo MT, Huber-Sannwald E, García ME, García HM, Aguado SGA. Selección de germoplasma de zacate navajita con diferente historial de uso en Jalisco, México. Tec Pecu Mex 2005;43(3):371-385.

48. Rubio AFA. Caracterización inicial de 59 ecotipos de zacate banderita (Bouteloua curtipendula (Michx.) Torr. En Calera, Zacatecas. Manejo Pastiz 1990;3(2):3-9.

49. Beltrán LS, García DCA, Hernández AJA, Loredo OC, Urrutia MJ, González ELA, et al. " Banderilla Diana" Bouteloua curtipendula (Michx.) Torr., nueva variedad de pasto para zonas áridas y semiáridas. Rev Mex Cienc Pecu 2013;4(2):217-221.

50. Beltrán LS, García DCA, Hernández AJA, Loredo OC, Urrutia MJ, González ELA, et al. "Navajita Cecilia" Bouteloua gracilis HBK (Lag.): Nueva variedad de pasto para zonas áridas y semiáridas. Rev Mex Cienc Pecu 2010;1(2):127-130.

51. Beltrán LS, García DCA, Loredo OC, Urrutia MJ, Hernández AJA, Gámez VHG. "Llorón Imperial", Eragrostis curvula (Schrad) Nees, variedad de pasto para zonas áridas y semiáridas. Rev Mex Cienc Pecu 2018;9(2):400-407.

52. Beltrán LS, García DCA, Loredo OC, Urrutia MJ, Hernández AJA, Gámez VHG. "Garrapata Hércules" Eragrostis superba (Peyr), variedad de pasto para zonas áridas y semiáridas. Rev Mex Cienc Pecu 2020;11(1):304-310. 
53. Beltrán LS, García DCA, Loredo OC, Urrutia MJ, Hernández AJA, Gámez VHG. "Titán" y "Regio", variedades de pasto buffel (Pennisetum ciliare) (L.) Link para zonas áridas y semiáridas. Rev Mex Cienc Pecu 2017;8(3):291-295.

54. Garza CRD. Producción de semilla de buffel Milenio. En: Tecnologías generadas, validadas o transferidas en los estados de Tamaulipas, San Luis Potosí, Coahuila y Nuevo León en el año 2009. SAGARPA-INIFAP-Campo Experimental Río Bravo. 2010. http://www.inifapcirne.gob.mx/Revistas/Archivos/FichasTecnologicas.pdf. Consultado 12 Oct, 2020.

55. Hernández RP, Cuéllar VEJ, Martínez VJ. Guía para el establecimiento y manejo de zacate buffel Zaragoza 115 para producción de semilla bajo riego. SAGARPA-INIFAPCampo Experimental Zaragoza. 2002. http://www.inifapcirne.gob.mx/Biblioteca/Publicaciones/228.pdf._Consultado 12 Oct, 2020.

56. Morales-Nieto C, Quero A, Le-Blanc O, Hernández A, Pérez J, González S. Caracterización de la diversidad del pasto nativo Bouteloua curtipendula Michx. Torr. mediante marcadores de AFLP. Agrociencia 2006;40(6):711-720.

57. Morales-Nieto CR, Quero A, Pérez J, Hernández A, Le-Blanc O. Caracterización morfológica de poblaciones nativas de pasto banderita [Bouteloua curtipendula (Michx.) Torr.] en México. Agrociencia 2008;42(7):767-775.

58. Morales NCR, Avendaño AC, Melgoza CA, Gil VK, Quero CA, Jurado GP, et al. Caracterización morfológica y molecular de poblaciones de pasto banderita (Bouteloua curtipendula) en Chihuahua, México. Rev Mex Cienc Pecu 2016;7(4):455-469.

59. Rubio-Aguirre FA, Villanueva JF, Sánchez RA. Comportamiento morfológico y productivo de "Colectas base" de gramíneas nativas e introducidas del altiplano de Zacatecas. Publicación especial. Núm. 22. Campo Experimental Zacatecas. CIRNOCINIFAP. Zacatecas. 2016. http://zacatecas.inifap.gob.mx/publicaciones/Publesp222016.pdf. Consultado 19 Sep, 2020.

60. Morales NCR, Quero CAR, Avendaño ACH. Caracterización de la diversidad nativa del zacate banderita [Bouteloua curtipendula (Michx.) Torr.], mediante su nivel de ploidía. Tec Pecu Méx 2007;45:263-278.

61. Comai L. The advantages and disadvantages of being polyploid. Nat Rev Genet 2005;6(11):836-846. 
62. Morales-Nieto CR, Álvarez A, Villarreal F, Corrales R, Pinedo A, Martínez SM. Phenotypic and genetic diversity of blue grama (Bouteloua gracilis) populations from Northern Mexico. Arid Land Res Manage 2019;34(1):83-98.

63. Avendaño-González M, Morales JF, Siqueiros ME. Genetic structure, phylogeography, and migration routes of Bouteloua gracilis (Kunth) Lag. ex Griffiths (Poaceae: Chloridoideae). Mol Phylogenetics Evol 2019;134:50-60.

64. Morales NCR, Madrid PL, Melgoza CA, Martínez SM, Jurado GP, Arévalo GS, et al. Análisis morfológico de la diversidad del pasto navajita [Bouteloua gracilis (Willd. ex Kunth) Lag. ex Steud.], en Chihuahua, México. Téc Pecu Méx 2009;47(3):245-256.

65. Morales NCR, Melgoza CA., Jurado GP, Martínez SM, Avendaño AC. Caracterización fenotípica y molecular de poblaciones de zacate punta blanca (Digitaria californica (Benth.) Henr.). Rev Mex Cienc Pecu 2012;3(2):171-184.

66. Morales-Nieto CR, Rivero O, Melgoza A, Jurado P, Martínez M. Caracterización morfológica y molecular de Leptochloa dubia (Poaceae) en Chihuahua, México. Polibotánica 2013;36:79-94.

67. Morales-Nieto CR, Avendaño AC, Melgoza CA, Martínez SM, Jurado GP. Caracterización morfológica y molecular de poblaciones de zacate tempranero (Setaria macrostachya Kunth) en Chihuahua, México. Phyton 2015;84(1):190-200.

68. SNICS. Servicio Nacional de Inspección y Certificación de Semillas. México. 2018. https://datos.gob.mx/busca/dataset/cnvv/resource/2651402e-8b42-411a-b23b22936176fb42. Consultado 25 Sep, 2020.

69. Eguiarte VJA, González SA. Producción de semilla y forraje de pastos tropicales en el Sur de Jalisco. Tec Pecu Mex 1995;33(2):105-111.

70. Sáenz-Flores E, Saucedo TRA, Morales NCR, Jurado GP, Lara MCR, Melgoza A, et al. Producción y calidad de semilla de pastos forrajeros como respuesta a la fertilización en Aldama, Chihuahua. Tecnocienc Chih 2015;9(2):111-119.

71. González DJR. Producción de semilla de dos variedades de zacate banderilla con diferentes densidades de siembra. Agraria 1988;4(2):137-145.

72. Velázquez-Martínez M, Santiago-Hernández F, Gámez-Vázquez HG, CervantesBecerra JF. Fertilización para la producción de semilla de pasto banderita (Bouteloua curtipendula) en condiciones de riego. En: Simposio "Manejo del pastoreo, Toral en el manejo de pastizales" y "60 aniversario del Rancho Experimental La Campana". Chihuahua, Chih. 2017:198-201. 
73. Beltrán LS, Loredo O C, García DCA, Hernández AJA, Urrutia MJ, Gámez VHG, et al. Llorón Imperial y Garrapata Hércules Nuevas Variedades de pastos para el altiplano de San Luis Potosí (Establecimiento y producción de semilla). INIFAP-CIRNE Campo Experimental San Luis. (Folleto Técnico No. 36). 2009.

74. Martín GO. Técnicas de refinamiento y recuperación de pastizales. 1a ed. San Miguel de Tucumán: Universidad Nacional de Tucumán. E-Book. ISBN 978-950-554-865-1. 2014.

75. Beltrán LS, Loredo OC. ¿Cuándo sembrar y cómo realizar una siembra de pastos? INIFAP-CIRNE-CE San Luis Potosí (Desplegable Técnico No. 2). 2005.

76. Sierra TJS, Ramírez GH, Gutiérrez RE. Paquete tecnológico para la siembra de pastos en los agostaderos de Chihuahua. INIFAP-CIRNOC Sitio Experimental La Campana. (Folleto Técnico No. 50). 2014.

77. Velázquez MM, Hernández GFJ, Cervantes BJF, Gámez VHG. Establecimiento de pastos nativos e introducidos en zonas semiáridas de México. INIFAP-CIRNE Campo Experimental San Luis. (Folleto para Productores No. 66). 2015.

78. Medina-Guillén R, Cantú-Silva I, González-Rodríguez H, Pando-Moreno M, Kubota T, Gómez-Meza MV. Efectos del rodillo aireador y el fuego en las propiedades físicas e hidrológicas del suelo en matorrales de Coahuila, México. Agrociencia 2017;51:471485.

79. Berlanga RCA. Uso del rodillo aereador para la rehabilitación de pastizales degradados. INIFAP-CIRNE-Campo Experimental Saltillo. (Desplegable Técnico No. 10). 2009.

80. Rivera SFJ, Pérez CJM, Montañez AMP, Lavandera BG. Uso del rodillo aireador en la restauración de pastizales en Agua Prieta, Sonora. Biotecnia 2017; XIX(3):23-28.

81. Olhagaray REC, García EG, Espinoza AJ. Influencia de dos sistemas de labranza mínima y tradicional en la producción de zacate buffel (Cenchrus ciliaris) empleando curvas a nivel con relación 1:1 en el suroeste de Coahuila, México. VI Simposio Internacional de Pastizales. Monterrey, N.L. 2009.

82. Unión Ganadera Regional de Jalisco. Establecimiento de zacate buffel. https://www.ugrj.org.mx/index2.php?option=com_content\&do_pdf. Consultado 29 Dic, 2020.

83. Castellanos-Villegas AE, Bravo CL, Koch WG, Llano J, López D et al. Impactos ecológicos por el uso del terreno en el funcionamiento de ecosistemas áridos y semiáridos. En: Molina FF, Van Devender RT, editores. Diversidad Biológica de Sonora. México. UNAM; 2009:157-186. 
84. CEPAB. Campo Experimental Pabellón. Agenda Técnica Agrícola Aguascalientes, México. SAGARPA-INIFAP; 2017.

85. Esqueda CMH, Carrillo R, Sosa M, Melgoza A, Royo MH, Jimenez J. Emergencia y sobrevivencia de gramíneas inoculadas con biofertilizantes en condiciones de invernadero. Tec Pecu Mex 2002;42(3):459-475.

86. Jurado-Guerra P, Sierra JS, Lara MC, Saucedo TR, Morales NC. Establishment of native grasses with biosolids in abandoned croplands in Chihuahua, Mexico. Appl Env Soil Sci 2013; http://dx.doi.org/10.1155/2013/573808.

87. Soussana JF, Tallec T, Blanfort V. Mitigating the greenhouse gas balance of ruminant production systems through carbon sequestration in grasslands. Animal 2010;4(3):334350 .

88. Henderson BB, Gerber PJ, Hilinski TE, Falcucci A, Ojima DS, Salvatore M, et al. Greenhouse gas mitigation potential of the world's grazing lands: modeling soil carbon and nitrogen fluxes of mitigation practices. Agric Ecosyst Environ 2015;207:91-100.

89. Medina-Roldán E, Arredondo JT, Huber-Sannwald E, Chapa L, Olalde V. Grazing effects of fungal root symbionts and carbon and nitrogen storage in a shortgrass steppe in Central Mexico. J Arid Environ 2008;72:546-556.

90. Jurado GP, Saucedo TRA, Morales NCR, Martínez SM. Carbono orgánico del suelo y su relación con la condición en pastizales y matorrales de Chihuahua. En: Paz F, Wong J, Bazán M, Saynes V editores. Estado actual del conocimiento del ciclo del carbono y sus interacciones en México: Síntesis a 2013. Texcoco, Estado de México. PMCColegio de Postgraduados-UACH-ITESM; 2014:62-69.

91. Schuman GE, Janzen HH, Herrick JE. Soil carbon dynamics and potential carbon sequestration by rangelands. Environ Pollut 2002;116:391-396.

92. Montaño NM, Ayala F, Bullock S, Briones O, García F, García R. Almacenes y flujos de carbono en ecosistemas áridos y semiáridos de México: Síntesis y perspectivas. Terra Latinoam 2016;34:39-59.

93. Zermeño-González A, Ríos EJA, Gil MJA, Cadena ZM, Villarreal QJA. Dinámica del flujo de carbono y de energía sobre un pastizal natural del norte de México. Bioagro 2011;23(1):1-13.

94. Yerena YJI, Jiménez PJ, Alanís RE, Aguirre COA, González TMA, Treviño GEJ. Dinámica de la captura de carbono en pastizales abandonados del noreste de México. Trop Subtrop Agroecosyst 2014;17;113-121. 
95. Delgado-Balbuena J, Arredondo JT, Loescher HW, Huber-Sannwald E, Chávez G, Luna $\mathrm{M}$, et al. Differences in plant cover and species composition of semiarid grassland communities of central Mexico and its effects on net ecosystem Exchange. Biogeosci 2013;10:4673-4690.

96. Delgado-Balbuena J, Arredondo JT, Loescher HW, Pineda MLF, Carbajal JN, Vargas R. Seasonal precipitation legacy effects determine the carbon balance of a semiarid grassland. JGR Biogeosci 2019;124:987-1000. DOI:https://10.1029/2018JG004799.

97. Linares-Fleites G, Tenorio MG, Torres E, Oroza HAA. Estimación del carbono orgánico en suelos por teledetección y modelos de regresión. Rev Latinoam Amb Cienc 2017;8(18):26-40.

98. Jurado GP, Juárez MM, Saucedo TRA, Morales NC, Martínez SM. Modelo ecológico de predicción de carbono en pastizales de Chihuahua. En: Paz F, Velázquez A, Rojo M editores. Estado actual del conocimiento del ciclo del carbono y sus interacciones en México: Síntesis a 2018. Texcoco, Estado de México. PMC-ITS; 2018:62-69.

99. Servicio Nacional de Inspección y Certificación de Semillas (SNICS). Catálogo Nacional de Variedades Vegetales. https://datastudio.google.com/u/0/reporting/5b7206ba-e190-48fe-969673523bfccf58/page/itBWB. Consultado 7 Jul, 2020. 